\title{
As Buffard As Possible
}

\author{
PAULINE LE BOULBA
}

I imagine choreographic pieces as friends, accomplices, places to roam, edges from which to (re)start. As food, in a way. With the series La Langue Brisée [The Broken Tongue], since 2015 I have been deploying life forms from the works of others, a procedure I started to call "réceptions performées" [performed receptions]: I conceive affective and gestural hypotheses from dance pieces I have seen. "As Buffard As Possible" tells my encounter and continued relationship with two works by French choreographer Alain Buffard (1960-2013): Dispositifs 3.1 (2001) and My Lunch with Anna (2005). But it also tells about my encounter with The Flying Lesbians, the first European rock band founded by lesbians in the 1970s, and how they too become my accomplices and affect my perception of Buffard's works. I ask them to accompany me on stage for La langue brisée (3), a piece that I presented in October 2017 at Centre National de Danse (CND) in Pantin, Paris. They answer that the group no longer exists and that they are too old now, though they agree to receive me at their homes. They tell me their stories. I record. I extend a fiction out of Buffard's works. I make an "affiliation-fiction". I identify myself with Heidi, the troubled figure of Dispositifs 3.1, stuck between learning and hacking, between imitation and betrayal. 
Je travaille sur La langue brisée (3), la dernière pièce d'un triptyque que j'ai amorcé en 2015 autour de ce que j'appelle des « réceptions performées » ou comment répondre à une danse en dansant. Certaines pièces chorégraphiques m'ont prise et j'ai eu envie de partager la manière dont j'en suis éprise. Spectatrice d'une danse qui m'a parlé, j'imagine, à mon tour, l'espace du plateau comme le lieu où je pourrais restituer ma réception. Usant de différents médiums (textes, gestes, actions, chansons, vidéos) afin de faire apparaître la pluralité de registres perceptifs qui constitue mon travail d'artiste et de chercheuse. J'échange beaucoup avec Laurent Pichaud qui danse, lui aussi, dans l'œuvre de quelqu'un d'autre. Celle de Deborah Hay. ${ }^{1}$ Il partage avec moi des morceaux d'un journal de bord de son voyage aux États-Unis auprès d'elle et dans ses archives à Austin, Texas. Je m'inspire de son format et notamment de la possibilité qu'offre le journal de bord pour rendre compte de différentes écritures (personnelle, réflexive, poétique, etc.) à travers une mise en page qui fait cohabiter des typographies comme autant de points de vue.

Mon dialogue avec Laurent Pichaud a donné lieu à une publication dans Le Journal des Laboratoires d'Aubervilliers en janvier 2018. J'en donne ici une version modifiée.

La langue brisée (3) raconte ma relation au travail du chorégraphe français Alain Buffard. ${ }^{2}$ Relation suscitée dans un premier temps par un sentiment de curiosité, puis par l'intuition qu'il y avait là une enquête à mener, un voyage à faire. C'est ce voyage dont je vais faire le récit. Je l'ai appelé « As Buffard As Possible ». Il s'origine dans deux ouvres du chorégraphe qui sont devenues pour moi des nourritures que j'ai mangées et qui ne s'épuisent jamais. Quand j'ai un creux je peux toujours y revenir.

La première œuvre est le film My Lunch with Anna, que Buffard a réalisé en 2005 et qui est composé d'une série de repas en compagnie de la chorégraphe américaine Anna Halprin. Celle-ci a joué un rôle déterminant dans son parcours. Dans les années 90, Buffard ne danse plus.

1 Laurent Pichaud est un danseur et chorégraphe français. Assistant de Deborah Hay depuis de nombreuses années, il a construit une relation amicale et professionnelle auprès d'elle. En 2017, il commence une thèse de recherche-création au département Danse de Paris 8 sous la direction d'Isabelle Launay et Julie Perrin, intitulée «Faire de l'in situ dans I'œuvre de Deborah Hay ». Alain Buffard était un danseur et chorégraphe français (1960-2013). 
Il s'occupe de la production du Quatuor Knust et dans ce cadre-ci rencontre Yvonne Rainer.

Yvonne Rainer était venue pour travailler avec les danseurs et j'ai réalisé que je commençais à passer bien plus de temps dans le studio que dans mon bureau. J'ai fini par faire partie intégrante du projet. Ça a tout atomisé dans ma tête après sept ans d'arrêt de danse. D'avoir Yvonne Rainer en face de moi, de pouvoir lui poser toutes les questions que je me posais depuis sept ans et qu'elle a résolues dans sa danse et en accord avec les gens avec qui elle travaille, de pouvoir évoquer les raisons pour lesquelles elle a arrêté de danser [...] m'a donné beaucoup de réponses sur le partage du pouvoir entre la danse et la chorégraphie, sur le minimalisme. Le minimalisme a été majeur mais jamais questionné en danse, quoi qu'on en dise. Or, en faire le moins pour montrer le plus, c'est bien l'angle d'attaque de mon travail. Et la personne détentrice de tout ça c'est Anna Halprin. ${ }^{3}$

C'est la première fois qu'il entend parler d'Anna Halprin. En faisant quelques recherches à son sujet, il découvre que celle-ci donne des ateliers pour des personnes atteintes d'un cancer ou touchées par le SIDA. Il participe à un grand workshop pour 150 personnes, qu'elle donne à Fribourg en Allemagne. À cette occasion, Halprin présente un solo en hommage à son grand-père. Elle a 75 ans. Elle porte un pyjama et des grosses boots et elle fait une sorte de danse russe, très physique, qui va impressionner Buffard. ${ }^{4}$ Puis il suivra un autre atelier auprès d'elle, en Californie, durant trois mois. À son retour, il créera son solo Good Boysorte d'autoportrait de l'artiste qui le fera connaître en tant que chorégraphe et à partir duquel il développera d'autres pièces.

Le film qu'il réalise revient sur leur rencontre et met surtout en avant la démarche chorégraphique d'Halprin. Le moment que je préfère c'est quand ils mangent un morceau de tomate au ralenti, en se regardant dans le fond des yeux.

À partir de ce film, j'ai proposé à mes grands-parents Alain \& Niquette Castellani de rejouer quelques scènes en version française. Ça faisait un

3 «Alain Buffard et ses dispositifs 》, interview avec Fabienne Arvers, in Les Inrocks, 28 mars 2001 - Archives Buffard, CND, Pantin.

4 II relate ses impressions dans «Critical Correspondance - Interview with Jennifer Monson », in Movement Research, 4 septembre 2006 - Archives Buffard, CND, Pantin. 
moment que je voulais partager un bout de ma thèse avec eux et comme ils sont plutôt joueurs, ce n'était pas difficile de les convaincre. Ils n'arrivaient pas à apprendre leur texte alors on cachait les feuilles et je filmais en plan resserré. Ils habitent dans une maison sur la côte Ouest de la Corse du Sud, en face de la mer. Ça pourrait ressembler à la Californie, lieu où vit Halprin et où Buffard a réalisé son film.

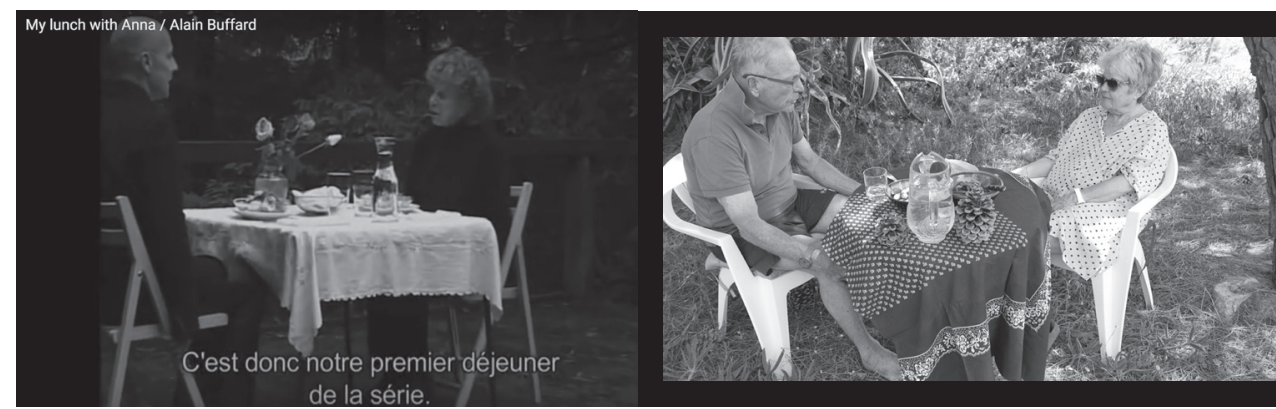

La deuxième œuvre que j'ai mangée et que j'associe à ce film c'est Dispositifs 3.1, une pièce de 2001 qui met en scène Buffard lui-même, les danseuses Anne Laurent et Claudia Triozzi et la critique de danse et théoricienne Laurence Louppe.

La présence d'Halprin flotte étrangement dans cette pièce. FantômeHalprin.

Si elle est tenue pour l'une des figures majeures de la modernité en danse, son travail se situe à mes yeux au-delà de cette catégorie ; notre rencontre a provoqué à mon retour le désir de revenir à la danse après une « cessation d'activité » d'une durée de 7 ans. Par la suite, je peux dire que son ombre a toujours plané sur l'ensemble de mes productions chorégraphiques. Pour autant, je ne pourrais pas dire que celles-ci s'inscrivent dans une filiation esthétique ou politique avec son œuvre - comme on l'entendrait d'un danseur sortant du moule d'un chorégraphe. ${ }^{5}$

Sa présence se fait aussi ressentir au regard des thématiques abordées dans la pièce, qui s'articulent principalement autour du dressage des corps (apprentissage de l'enfant, apprentissage du·de la danseur·se, etc.). 
Anna Halprin travaille depuis les années 70 à déconstruire par la danse les normes corporelles et sociales.

Sur le plateau les quatre interprètes portent tou.t.e.s une perruque blond platine qui leur recouvre le visage, en clin d'œil à Heidi, la petite fille du conte pour enfants qui vit des aventures dans la montagne. Buffard s'était inspiré du film des artistes Mike Kelley et Paul McCarthy, qui revisite le conte mais en version trash. Buffard propose à son tour une Heidi troublée et troublante, coincée entre apprentissage et piratage. Ça dérape, le cadre s'altère. Le tablier laisse voir le dos, la partie inconsciente du corps, expliquait Buffard.

Qu'est-ce que ce serait, de laisser son inconscient parler en tant que spectatrice?

Pour Dispositifs 3.1, Buffard avait établi une constellation de références plutôt affiliées à une culture underground américaine des années 60-70 et notamment des références gay mais pas seulement. Je les branche à d'autres références issues de cette époque-là, que je connais déjà ou que je croise sur mon chemin. Face à elles, d'autres apparaissent. Je dessine une cartographie de ma réception.

Heidi devient ma liaison. Elle me guide. Sorcière, fantôme, idea, esprit, souffle, petite fille, hair pur.

Désormais, tout ce que je trouve sur mon chemin devient signe envoyé de la planète 3.1. 

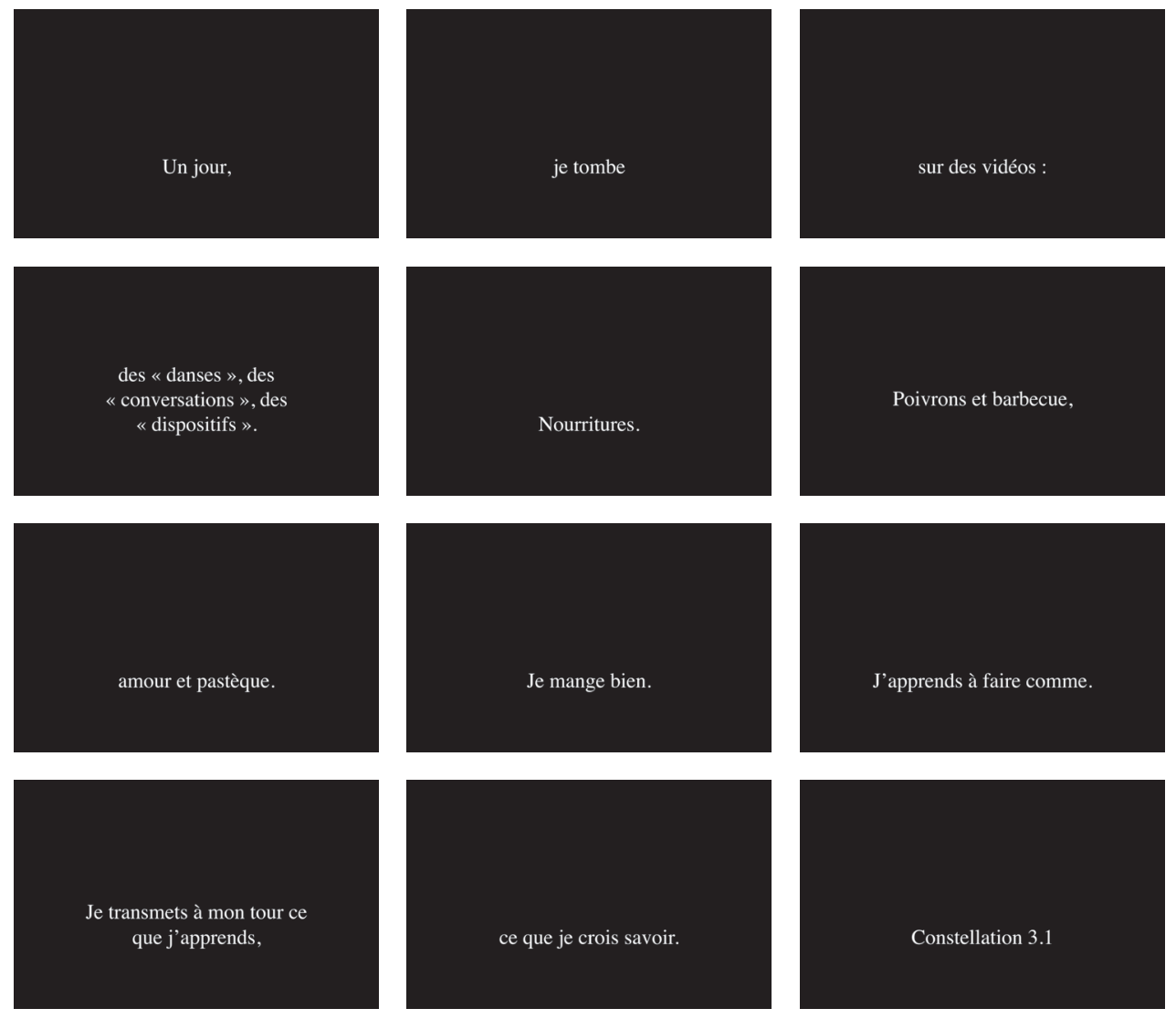
Depuis presque deux ans, je marche dans le travail du chorégraphe Alain Buffard. Je marche d'abord dans la pièce Dispositifs 3.1, puis dans le film My Lunch with Anna. J'aime les penser comme les faces d'une feuille de laitue géante sur laquelle je marche, je transpire, je fais la sieste, je ralentis mon regard.

Je n'ai pas connu personnellement Alain Buffard, la seule fois où je l'ai croisé, je venais de commencer ma thèse sur « la critique performative en danse », sorte de MacGuffin de mon enquête universitaire, artistique et personnelle. Dispositifs 3.1 figurait dans mon corpus, mais ce jour-là, je n'ai pas osé lui parler. Il disparaissait quelques jours plus tard en décembre 2013.

samedi 12 août 2017 // Paris > Köln > Nettersheim

Il est 6 h du matin, le train est déjà bien rempli. J'ai dormi cinq heures cette nuit et je ne parviens pas à somnoler comme le font mes voisin.e.s. Paula fait le voyage avec moi. J'ai organisé ce rdv depuis quelques mois déjà.

En décembre 2016, dans un appartement berlinois, je découvre par hasard un CD des Flying Lesbians, le premier groupe de rock fondé par des lesbiennes en Europe. Elles viennent d'Allemagne de l'Ouest et ont enregistré un album en 1975. Leur musique me plait beaucoup et, après quelques recherches sur internet, je trouve un contact. ${ }^{6}$ Assez spontanément, je leur propose de venir interpréter des chansons pour La langue brisée (3) - une performance qui s'appuie sur ma réception du travail d'Alain Buffard - et que je présenterai en octobre 2017 au Centre National de la Danse. Monika, Cillie et Danielle me répondent chacune. Elles m'expliquent que le groupe ne joue plus et que deux des sept Flying Lesbians ne sont malheureusement plus là.

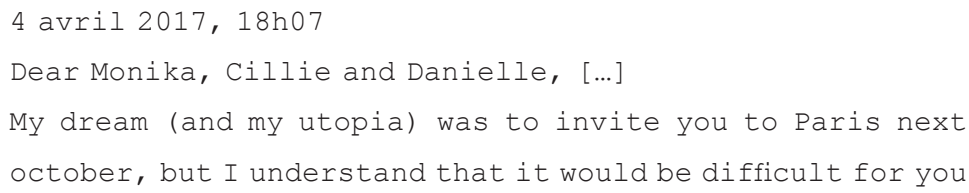


to get together and come to France. So I propose to come to see each one of you separately, to talk about the "Flying Lesbians" story and perhaps make a documentary on the group. I think it's very important today to keep the memory of the group alive and to share your stories in France and Germany!

The best time for me to come visit you would be in August. [...] Unfortunately I don't speak german at all [...]

With sisterly greetings, Pauline.

De ce mail, deux Flying Lesbians ont répondu favorablement à ma proposition de venir les rencontrer. Ce sont elles que nous partons retrouver. D’abord Monika à côté de Köln, puis dans quelques jours, Cillie près de Berlin.

Depuis Köln, nous prenons d'autres trains pour nous rendre à Nettersheim, là où vit Monika. Elle vient nous chercher à la gare. Le temps est automnal. Elle nous accueille chaleureusement. J'ai un sourire jusqu'aux oreilles. Elle nous dit avec malice que nous sommes dans «la capitale des lesbiennes », qu'il y a eu ici une vague de femmes qui sont venues s'installer en couple ou en communauté dans cette campagne verdoyante. Ici se trouvent des autels à l'effigie de matrones, déesses de la fertilité, qui datent du ler siècle. L'ambiance est mystique. La chienne de Monika nous attend dans la vaste salle à manger/cuisine, le frisbee dans la gueule. La maison de Monika est attenante à d'autres maisons, celle de sa compagne Claudia, et celles de deux amies. Monika a acheté des gâteaux pour nous.

Une partie de son histoire commence. Je filme et j'enregistre. Elle revient sur son arrivée à Berlin Ouest quand elle avait 22 ans, en tant que journaliste et chanteuse dans divers groupes. Jusqu'au jour où elle rencontra Cillie et les autres jeunes femmes. Elle nous décrit I'ambiance des concerts et nous montre un album photo. En noir et blanc.

La première arrive comme par magie. 


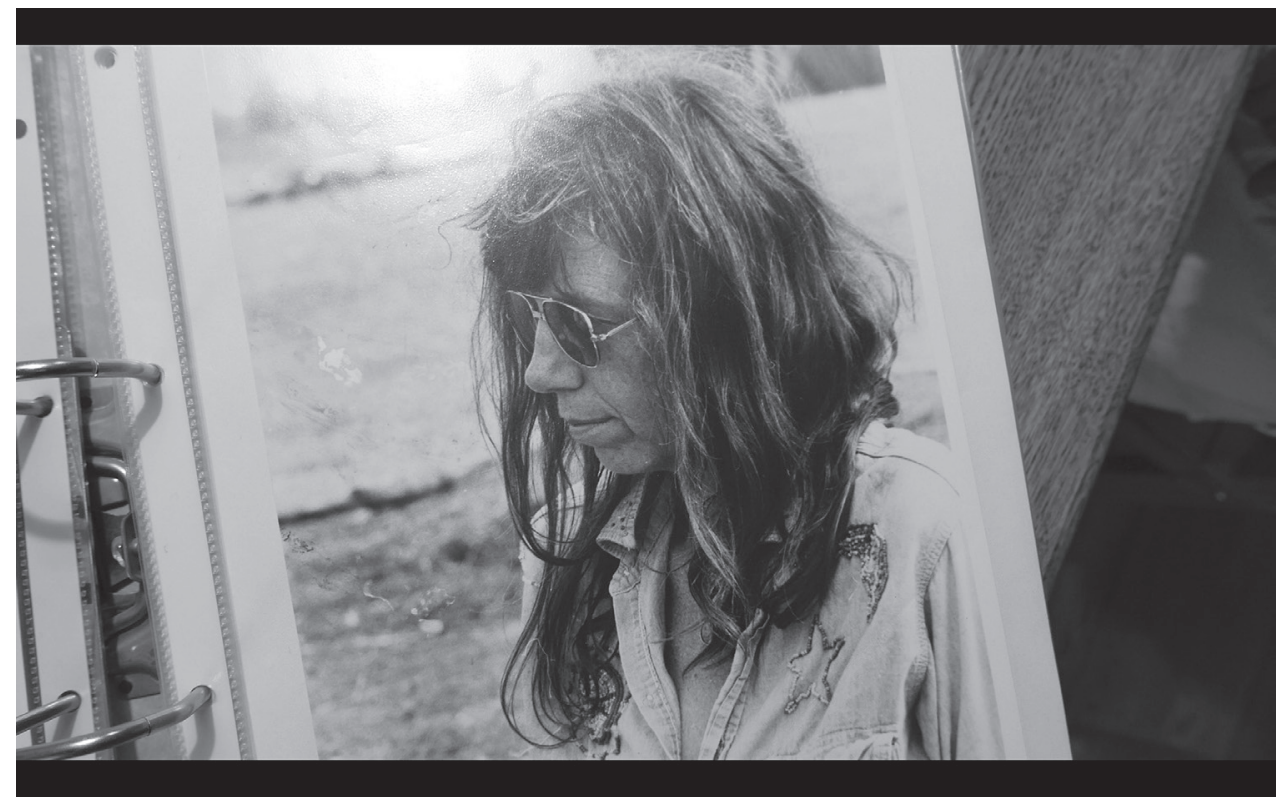

UN PORTRAIT DE JILL JOHNSTON AU FESTIVAL FEMØ À COPENHAGUE EN 1974, OÙ LES FLYING LESBIANS ONT JOUÉ À L'ÉPOQUE. AU STYLO EST ÉCRIT LESBIAN NATION.?

Jill Johnston fait partie de ces fantômes avec lesquels je m'imagine parfois en pleine conversation.

Je raconte à Monika que Jill Johnston était une critique de danse aux Etats-Unis dans les années 6o, à New York plus précisément. Qu'elle a fait un travail considérable pour soutenir le mouvement des avantgardes artistiques et notamment les artistes chorégraphiques (Yvonne Rainer, Deborah Hay, Steve Paxton...), et qu'elle danse dans un film d'Andy Warhol. Elle a écrit principalement dans The Village Voice et a compilé certains de ses articles dans son livre Marmalade Me (1971). On y lit au fil de ses chroniques un ton libre et une soif d'échapper aux catégories normatives. Elle a un parcours atypique, puisqu'elle cesse son activité de critique au moment où elle rejoint les mouvements activistes féministes. Elle organise pour l'occasion une table-ronde à New York, en 1969, intitulée symboliquement " The disintegration of a critic ». Son drop out se transforme en coming out. Elle publiera, en 1973, Lesbian Nation et poursuivra une activité littéraire et militante. Son parcours est, 
dans mon imaginaire, associé à d'autres figures de critiques féminines qui effectuèrent elles-aussi un retrait du métier de critique, du moins un déplacement radical. À la fin des années 60 en Italie, la critique Carla Lonzi décide de quitter le milieu de l'art. ${ }^{8}$ Elle publie, en 1969, Autoritratto qui s'avère être un montage fabriqué à partir d'entretiens avec des artistes et dans lequel sa propre voix démonte progressivement la figure $\mathrm{du} / \mathrm{de}$ la critique. Un autoportrait en forme d'autocritique sur sa pratique. Elle aussi rejoint un féminisme militant et sera fondatrice du collectif Rivolta Femminile. Influencée par la critique post-structuraliste de Barthes (« La mort de l'auteur » paraît en 1968) ainsi que celle de Susan Sontag, qui défend un projet critique « contre l'interprétation » (1964), elle écrit dans l'introduction de son ouvrage : « À un certain moment, j'ai ressenti l'œuvre d'art comme une possibilité de rencontre, comme une invitation à participer, que les artistes adressent directement à chacun de nous. Un geste auquel, il m'a semblé, je ne pouvais pas répondre de façon professionnelle ». La polyphonie des voix participe à l'invention d'un nouveau corps critique et prend part à tout un courant littéraire et esthétique porté par un désir de se défaire des normes langagières et esthétiques. Je me demande si Carla Lonzi a rencontré Jill Johnston, et si elle écoutait la musique des Flying Lesbians.

Je pense aussi à la critique de danse Laurence Louppe - figure associée à bon nombre d'artistes chorégraphiques français par l'influence qu'elle aura eu auprès d'eux - qui se prêtera au jeu du travestissement. Dans la pièce d'Alain Buffard Dispositifs 3.1, elle joue de sa posture de théoricienne d'art, qui se moque d'elle-même. Affublée d'une perruque blonde, et tout en se maquillant négligemment, elle porte d'une voix criarde un discours ironique sur l'art pour finir dans un carton duquel elle sort énervée en criant « Fuck you white cube! ».

Et puis je pense à Isabelle Ginot, qui dirige ma thèse de doctorat à Paris 8, et à ses articles sur Mark Tompkins, dans lesquels elle interroge sa posture de critique, de femme, de spectatrice... « Comment tous nos corps, nos mots, nos sentirs et nos gestes sont envahis par les corps des autres, leurs mots, leurs sentirs et leurs gestes $\gg{ }^{9}$

Voir à ce propos l'intervention d'Elisabeth Lebovici, « Carla Lonzi, Les Parleuses, le silence », lors de la journée d'étude à l'occasion de la parution française de Carla Lonzi, Autoportrait (JRP Ringier, coll. «Lectures de la Maison Rouge», 2012), conçue et organisée par Giovanna Zapperi avec Travelling féministe, en collaboration avec I'Association des amis de la Maison Rouge. http://www.dailymotion.com/video/xz3ilz.

9 Ginot Isabelle, « Mark Tompkins, Livin' is deadly », in Art Press «Medium Danse», numéro spécial, novembre 2002, pp. 56-59, et aussi «Les croyances suspendues », in La danse en solo. Une figure singulière de la modernité, dir. Claire Rousier, CND, coll. Recherches, Pantin, 2002. 
Ma caméra n'a plus de batterie, j'ai déjà filmé trois heures. Un signe peut-être. La conversation se poursuit, nous allons sur des terrains plus personnels.

Elle me demande ce que je vais faire de tout ça. Je lui dis que je ne sais pas. Que je suis venue pour mon enquête. Que Buffard m'y a conduite d'une certaine manière.

Ici je vacille. La voix grave et chaude de Monika m'emporte vers d'autres horizons, me transporte vers d'autres désirs. Plus personnels peut-être. J'éprouve concrètement l'œuvre de Buffard comme un bord duquel partir. Je sens que je m'en éloigne et bizarrement je ne me suis jamais sentie aussi proche d'elle, de lui. Nager vers le large, rejoindre un récif. Abandonner l'œuvre, en retrouver d'autres, en inventer de nouvelles. Je m'émancipe loin des miens dans une autre langue.

En partant, je lui fais signe de la main pendant quelques secondes, elle fait de même dans sa voiture et s'en va. Séquenceémotion. Larmes qui coulent dans mes veines.

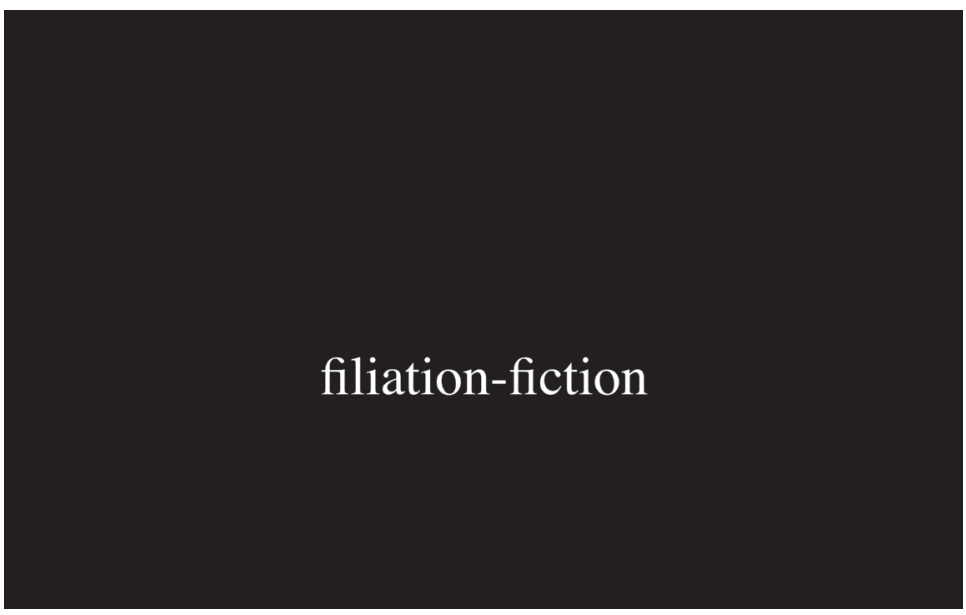

dimanche 13 août 2017 // Köln

Flâner à Köln. Manger un berliner. Prendre des photos. Passer par les sites touristiques. Entrer dans la cathédrale pour en ressortir immédiatement. Trop de monde. Parier une bière avec Paula que 
c'est la cathédrale la plus haute d'Europe. Perdre son pari. Fuir les touristes pour se retrouver dans un parc le long du Rhin. Lever la tête vers le ciel et voir un avion passer. Penser à celui qu'on prendra demain pour retrouver Cillie. Manger un bretzel. Goûter la Kölsch. Marcher encore. Manger une saucisse. Penser à toutes les saucisses, tous les bretzel et tous les Berliner mangés par les touristes aujourd'hui. Trouver ça dégueulasse.

J'ai croisé dans la rue un homme en tenue bavaroise. Ça m'a fait penser à la gamine Heidi et ses tenues traditionnelles. J'adore moi aussi porter la perruque. À l'envers. Ne plus rien voir ou presque. Dans Dispositifs 3.1, Laurence Louppe, les yeux bandés, sur les genoux de Claudia Triozzi, répète des phrases en allemand que lui dicte Anne Laurent. Une autre version d'Heidi: «Heidi est jeune, Heidi est saine, Heidi est émotive, elle aime les plantes et les animaux, les fleurs lui tirent les larmes, la montagne lui sourit et les chèvres lui disent bonjour le vent caresse sa chevelure blonde le ruissellement de la rivière la soulage, elle retrouve toujours le chemin de sa maison, Heidi aime les perruques, Grand-Père dit : Heidi est trop innocente pour comprendre l'ornement, Heidi sent bon l'odeur du foin, Grand père dit : Heidi n'a pas besoin de parfum, Heidi est docile, Heidi fait toujours plaisir à grand père, Heidi est très docile».

Où est Heidi aujourd'hui ? Que fait-elle ? À quoi pense-t-elle ? Depuis Dispositifs 3.1 quel a été son chemin?

\section{vendredi 18 août}

\section{BERLIN - MICHENDORF}

Cillie nous attend à la gare. Il y a un malentendu, je pensais que c'était sa compagne Cristina qui venait nous chercher puis au bout de deux minutes, je comprends que c'est Cillie. Je suis très gênée de ne pas l'avoir reconnue. Elle ne le prend pas mal. Nous montons dans sa voiture puis nous arrivons chez elle. Une maison qui donne sur un vaste champ. Cillie nous fait visiter le grand jardin puis nous rejoignons Cristina qui s'occupe des chèvres. Une d'elles vient d'accoucher de deux petits. Ils ont quatre jours et sont en pleine forme. Très sociables, ils sautent à nos pieds et nous les caressons comme des chiens. Nous bavardons longuement. Le discours de Cillie est différent de celui de Monika. Plus universitaire peut-être. Quand elle a fondé le groupe, elle commençait un doctorat. Sa bourse de 
thèse fut une aide précieuse au financement des activités des Flying Lesbians. Cristina nous rejoint et accepte d'être filmée à son tour. Avant cela, elle nous fait une démonstration de son drone dans le jardin. La scène est surréaliste, le drone dirigé par cette sexagénaireréalisatrice-fermière-fromagère, les chèvres au loin qui agitent leurs cloches et moi qui filme. Après un atterrissage réussi, nous goutons ses fromages de chèvre, Cristina est modeste, nous découvrons que les Flying Lesbians, c'était son idée. C'est elle qui a suggéré à Cillie de former un groupe de rock composé de femmes. C'est elle aussi qui a diffusé une annonce à la radio pour trouver des musiciennes. Quelques jours plus tard, le groupe était formé, les répétitions avaient lieu dans une maison à Kreutzberg - un quartier de Berlin jusqu'au premier concert en 1974 dans le hall de I'Université Technique de Berlin qui rassembla plus de 2000 femmes.

Cillie nous montre une photo d'elle en noir et blanc dans une baignoire, les cheveux et le buste mouillés, elle doit avoir une vingtaine d'années. «C'est là qu'on écrivait les chansons » dit-elle en souriant.

Alain Buffard était parti filmer Anna Halprin en Californie, entre quête identitaire et quête artistique - c'est ce que je me raconte. Pour moi, l'Allemagne devient un terrain de je(u). Les Flying Lesbians m'apprennent elles aussi à comprendre un peu mieux ce que je fais, ce que je veux faire ou ce que je ne veux pas faire. Elles deviennent mes complices, mes sœurs. Monika et Cillie, chacune avec leurs mots, me renseignent sur le militantisme lesbien des années 70 en Allemagne de l'Ouest, elles me dévoilent les contradictions des actions collectives, elles me partagent un peu de ce qu'elles étaient à 20 ans, elles me font goûter des pâtisseries ou du fromage de chèvre, on discute " convergence des luttes » en 2017. J'imagine parfois qu'à travers leurs récits, c'est Heidi qui me parle. Dans ces campagnes allemandes, mon travail prend des voies inattendues, on dirait qu'il entend des voix aussi. Kazuo Ôno, qui occupe une place dans ma thèse, raconte que La Argentina l'a appelé, un jour, alors qu'il regardait un tableau de peinture abstraite. Qu'elle lui a demandé de danser pour/avec elle. C'est ce qu'il fait en 1977, avec son solo Admiring la Argentina. À mon tour, je suis prise par les œuvres que je regarde. Heidi m'interpelle, je suis des pistes, des signes. Les signes, écrit Vinciane Despret, "sont toujours interpellatifs. Interpeller, le dictionnaire nous le précise, non seulement renvoie au fait 
d'appeler pour demander quelque chose, pour sommer de répondre, mais également à celui de causer une attention particulière, voire à contraindre à regarder. [...] Si le contenu informationnel d'un signe peut être en attente d'interprétation, ce n'est pas ce contenu qui importe, c'est le fait qu'il soit adressé, et que cette adresse pose chez celui qui le capte ou le reçoit, une question : qu'est-ce que je fais à partir de cela ? [...] C'est cela que fait le signe : il met au travail $\gg .^{10}$

10 «Penser par les effets. Des morts équivoques », in Études sur les morts. Thanatologie, n. ${ }^{\circ 142,}$ 2012/2, en ligne: http://www.vincianedespret.be/wp-content/uploads/Despret-E2-penserpar-les-effets-copie.pdf. 


\section{Qu'est-ce qu'elle me ferait faire Heidi ?}


En rentrant de mon voyage, je mêle dans un récit Heidi et les Flying Lesbians, je demande à mes grands-parents de porter la perruque. J'essaye de troubler une quelconque origine. De faire circuler une référence qui passe de tête en tête. Par capillarité.

J'écris des chansons sur/pour Heidi. J'apprends des séquences de Dispositifs 3.1, j'imite et je tords.

Deux danses composent La langue brisée (3), une reprise solitaire d'une séquence dite du défilé où les quatre interprètes de Dispositifs 3.1 se secouent certaines parties du corps sur une chanson de Patti Smith. Je reprends la séquence à l'identique comme pour retraverser encore une fois la pièce originale et la faire goûter aux spectateur-ice's. La deuxième séquence qui reprendra le même parcours dans l'espace sera un négatif de cette danse injonctive. Ici je joue des codes du défilé en sapant les codes féminins. Une danse $100 \%$ transpiration. Mon visage n'est plus caché par la perruque mais grimace. Je reste ancrée dans le sol et propose un voguing-krump autodidacte et improvisé. La danse d'Heidi telle que je me la raconte, telle que je la traduis.

Plus tard, je reçois une invitation du Centre d'Art La Terrasse à Nanterre pour présenter quelque chose dans le cadre du cinquantenaire de mai 68 en mars 2018.

Je propose à une chorale féministe basée à Paris - A feminist choir - de venir chanter une des chansons des Flying Lesbians. ${ }^{11}$ Elles en choisissent une de 1975, Frauen Kommt Her qu'elles traduisent en français et arrangent avec le musicien Gérald Kurdian. J'apprends la chanson avec elles et nous la chanterons pour l'occasion. J'avais oublié ce que procure la puissance d'un groupe qui chante. J'avais oublié la force du souffle - du sien et de celui des autres - qui longe la colonne vertébrale. La chanson est désormais à elles. Elles la chantent à présent dès que la chorale est invitée quelque part. Prolongement des Flying Lesbians. 


\section{RÉFÉRENCES}

BUFFARD, Alain (2001), « Alain Buffard et ses dispositifs », interview avec Fabienne Arvers, Les Inrocks, Archives Buffard, Pantin, CND.

- (2003), «Quelques notes sur Anna Halprin. Pour un projet de film vidéo 2004 », Archives Buffard, Pantin, CND.

- (2006), « Critical Correspondance - Interview with Jennifer Monson », in Movement Research, Archives Buffard, Pantin, CND.

DESPRET, Vinciane (2012), « Penser par les effets. Des morts équivoques », in Études sur les morts. Thanatologie, n. ${ }^{\circ}$ 142, en ligne: http://www.vincianedespret.be/wp-content/uploads/Despret-E2-penser-par-les-effets-copie.pdf.

GINOT, Isabelle (2002a), « Mark Tompkins, Livin' is deadly », in Art Press « Medium Danse », numéro spécial, pp. 56-59.

- (2002b), « Les croyances suspendues », in Claire Rousier (dir.) La danse en solo. Une figure singulière de la modernité, Pantin, CND, coll. Recherches, cité d'après la version électronique publiée sur le site Paris 8 Danse : www.danse.univ-paris8.fr.

FLYING LESBIANS, http://flying-lesbians.de/.

Johnston, Jill (1971), Marmalade Me, New York, E. P. Dutton \& Co., Inc.

- (1973), Lesbian Nation. The Feminist Solution, New York, A Touchstone Book, Simon and Schuster.

LE BOUlBA, Pauline (2018), As Buffard As Possible, Lecture-augmentée, Nanterre, Centre d'Art la Terrasse, https:// www.youtube.com/watch?v=7R-32uYqPSI.

LE BOVIC I, Elisabeth (2012), « Carla Lonzi, Les Parleuses, le silence », Intervention orale à la Maison Rouge, Paris, http://www.dailymotion.com/video/xzzilz.

LONZI, Carla (2012), Autoportrait, Paris, JRP Ringier, La Maison Rouge.

\section{ILLUSTRATIONS @ PAULINE LE BOULBA}

1. «My lunch with Anna » (capture d'écran, vidéo YouTube)

2. Alain et Niquette

3. Jill Johnston au festival Femø à Copenhague en 1974

4-17. Diapositifs image-texte

\section{PAULINE LE BOULBA}

É artista e investigadora em dança. O seu trabalho inscreve-se numa reflexão em torno dos discursos sobre/da dança, tentando propor novas formas de percepcionar as obras, de com elas dialogar e/ou a partir delas dançar. Como respostas críticas às peças com as quais entra em contacto, imagina outros objectos e performances (ensaios, poemas, rap). A sua investigação artística articula-se no campo reflexivo da tese de doutoramento que desenvolve no Departamento de Dança da Universidade de Paris-8, sob a direcção de Isabelle Ginot e com o título Les danses des spectat:eur.rice·s. Réceptions performées et critiques affectées (As danças da.o.s espectador.a.e.s. Recepções performadas e críticas afectadas) (título provisório). 